\title{
An Analysis of MOOC Discussion Forum Interactions from the Most Active Users
}

\author{
Jian-Syuan Wong, Bart Pursel, Anna Divinsky, and Bernard J. Jansen \\ The Pennsylvania State University, University Park, PA, USA \\ \{jxw477, bkp10, axd289\}@psu.edu, jjansen@acm.org
}

\begin{abstract}
Many massive open online courses (MOOCs) offer mainly videobased lectures, which limits the opportunity for interactions and communications among students and instructors. Thus, the discussion forums of MOOC become indispensable in providing a platform for facilitating interactions and communications. In this research, discussion forum users who continually and actively participate in the forum discussions throughout the course are identified. We then employ different measures for evaluating whether those active users have more influence on overall forum activities. We further analyze forum votes, both positive and negative, on posts and comments to verify if active users make positive contributions to the course conversations. Based the result of analysis, users who constantly participate in forum discussions are identified as statistically more influential users, and these users also produce a positive effect on the discussions. Implications for MOOC student engagement and retention are discussed.
\end{abstract}

Keywords: MOOC $\cdot$ Learning forum $\cdot$ Online learning $\cdot$ Distance education

\section{Introduction}

MOOCs have become a popular and significant source of distance-education due to the flexibility of course access and varied topics of courses. With these benefits, there are million students who enroll in one or more MOOCs. Since MOOCs are typically offered with only video-based lectures from are not co-located with the professor, students usually lack the opportunity to have interactions with other students and the instructor relative to traditional resident courses. Therefore, technology-based interactions, such as blogs and forums, are introduced as potential solutions. A discussion forum offers a platform for asynchronous communications that facilitates interactions and communications among students and instructors, and it also helps students build a learning community within the MOOC. Prior work has noted that discussions among peers help students improve their learning performance [1]. Additionally, discussions in a forum provide useful information for instructors to monitor course progress [2]. Therefore, discussion forums are widely adopted by MOOCs for interaction enhancement.

Due to the importance of the discussion forum to MOOCs, we conduct analyses to explore the interactions and communications in a MOOC forum. Forum users who 
actively participate in discussions are first identified based on their continuous participation, the number of replies they receive, and the number of responses they make. In order to verify whether active users are also influential users in the discussion forum, different measures of forum threads made by these active users are studied and compared with typical (i.e., non-active) forum users. Moreover, we analyze votes, both positive and negative, of posts and comments from active users to evaluate if active users make positive contributions to the MOOC forum.

\section{Related Work}

Anderson [3] discusses the factors of successful forums, as well as the measurements and limitations for online learning. Social network analysis is commonly applied to analyze the interactions on learning forums [4]. Dropout behavior is identified as relevant to sentiment of students' forum posts based on sentiment analysis of MOOC forums discussions [5]. Hauang et al. [6] studied the behavior of users who make great contributions across different MOOC forums offered by Coursera. The results indicate half of these 'superposters' are males, aged between 20 and 34. In addition, superposters commonly have better learning performance than the average forum users. Furthermore, these superposters generally have similar behaviors across different MOCCs that they take. However, there has been limited study of the effect of active posters on the learning environment of discussion forums.

\section{MOOC Forum}

We conceptualize a MOOC forum as composed of three types of hierarchical interactions, which are: A thread is created for initiating a new discussion. A post is a message for replying to a thread. A comment is a message used to reply to a post. In the 7-weeks course period for the MOOC used in this research, there are 8,169 threads, 21,434 posts, and 22,166 comments created by 7,389 forum users.

In order to provide insightful understanding of forum activities, we focus on user engagements. If a user initiates at least a new thread or makes a comment/post in a particular week, we consider this user to participate in the discussion for that week. Users' participations in each week are accumulated to study the number of weeks each user has engaged in the MOOC forum, and all of the forum users are divided into 7 groups based on weeks of involvements, from 1 to 7 weeks.

Table 1 denotes the number of weeks forum users participated in, and the average replies these users received and made during each week. For those users who participated in forum discussion for the entire course, the average replies they received and made are 80.31 and 113.00 , respectively. So, these active users contributed more than others react to their contributions. Furthermore, the number of replies made is 1.41 times higher than replies received for these users, which implies that these users are dedicated to making responses to other users. For users who participate for 6 weeks, they also have the similar behavior but with fewer replies received and made. 
Table 1. Number of users and average replies they receive/create based on weeks of participation

\begin{tabular}{c|c|c|c}
$\begin{array}{c}\text { Weeks of } \\
\text { Participation }\end{array}$ & \# Users & $\begin{array}{c}\text { Average Replies } \\
\text { Receive }\end{array}$ & $\begin{array}{c}\text { Average Replies } \\
\text { Create }\end{array}$ \\
\hline 1 & 5,021 & 1.84 & 1.75 \\
\hline 2 & 1,225 & 5.08 & 5.30 \\
\hline 3 & 538 & 9.88 & 10.31 \\
\hline 4 & 252 & 15.58 & 16.52 \\
\hline 5 & 174 & 24.10 & 24.28 \\
\hline 6 & 98 & 35.08 & 53.22 \\
\hline 7 & 81 & 80.31 & 113.00
\end{tabular}

Therefore, users who participate in the forum discussion for 6 and 7 weeks can be considered as active users not only for the higher number of replies received and made but also for the continuous participation. Non-active users are classified as typical users who participated 5 weeks or less.

\section{$4 \quad$ Research Question}

In order to evaluate whether the active users actually play significant roles in forum discussions, the first research question is do active users have more influence than typical users on the forum conversation? In addition, it is important to discover whether these active users have a positive or negative effect on the forum discussion once they are identified as influential users. Therefore, the second research question is do active users generally make a positive contribution to the MOOC forum? By answering these two research question, we are able to assess whether actives users are central to the MOOC discussion forum.

\section{$5 \quad$ Method}

Analysis for Research Question 1. For evaluating whether active users are more influential in forum discussion than typical users, three analyses are conducted based on forum threads, since a thread initiates a new discussion topic among forum users. Three measures of forum threads are used for the comparisons. 1. Number of times a thread is read by users (views of a thread). 2. Amount of replies, both posts and comments, a thread receives (replies of a thread). 3. How long does the discussions continue within a thread (duration of a thread). The number of times a thread is viewed indicates whether a discussion topic is widely captivating among students. More views of a thread means the content/idea behind this thread can be broadly expressed, becoming more influential. The number of replies made for the discussions within a thread implies user involvement on this discussion topic. More replies created for a thread denotes that the content/idea of this thread is able to facilitate user participation. Lastly, by assessing how long a conversation lasts in a thread, whether an initiated topic is continuously discussed by users, can be identified. If the discussion in a thread continues for a long period, it could indicate an influential or engaging topic. 
Analysis for Research Question 2. In order to answer whether active users generally make positive contributions to the MOOC forum, votes of posts and comments can be utilized for the evaluations. Forum users are allowed to give either a positive or negative vote to a post or comment based on the content. A post or comment that receives more positive votes implies the content is helpful and beneficial to other users; instead if a post or comment has more negative votes, it denotes that the content might be improper or irrelevant to the discussion topic. We can consider the posts or comments that receive more positive votes to have a positive contribution to the forum discussions, and the ones with more negative votes more likely to lead to negative impacts.

\section{$6 \quad$ Result and Discussion}

\subsection{Are Active Users More Influential?}

View of threads. For the 7 weeks of discussion, there were 828 threads created by active users and 7,370 threads created by typical users. The average views of threads created by active users and typical users are 126.14 and 53.93 times, respectively for overall discussions. As shown in Figure 1(a), the average views active users received for their threads are higher than typical users from week 1 through week 7 . In addition, an increasing trend can be observed from week 1 to week 4 for both groups of users, and the only drops happened in week 5 and 7.

Reply of Thread. Average replies within a thread made by active users received for the overall course period is 12.96 compared to 5.61 for typical users. Figure 1(b) illustrates the average replies for threads made by both types of users during the 7 weeks. Figure 1(b) shows that threads made by active users received more replies than the ones created by typical users in all 7 weeks. Furthermore, a similar trend can be seen in Figure 1(a), with an increasing trend of replies from week 1 to week 4, but average replies decrease both on week 5 and 7 . There are four weeks in which average replies of threads made by active users are twice that of typical users, which are weeks 1,2 , 5 , and 6 .

Duration of Thread. Duration of a thread is calculated by the time of last reply subtracted from the time a thread is initiated, with the difference rounded up to a day. The average duration of threads created by active users for the entire course is 3.89 days, which is 1.76 days more than typical users. In addition, Figure 1(c) shows the differences between active users and typical users for all 7 weeks for thread duration. Threads of active users have higher duration through the overall course, relative to typical users. The largest difference occurs for week 1 threads, which is 7.28 days for active users compared to 2.24 days for typical users. As shown in Figure 1(c), the duration of threads made by active users decrease weekly from 7.28 days in week 1 to 1.36 days in week 7 . However, they are always longer than threads of typical users. 


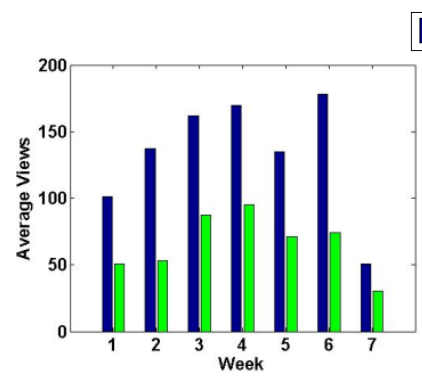

(a) Avg. views of threads

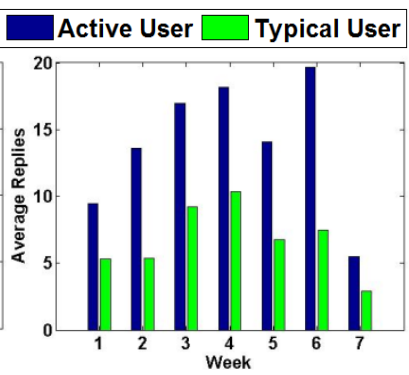

(b) Avg. replies of threads

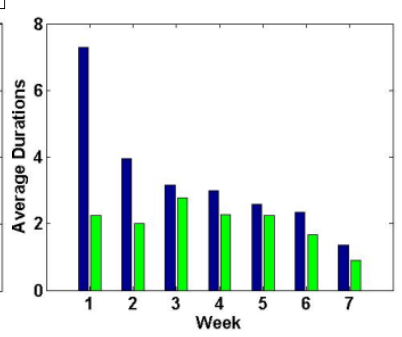

(c) Avg. durations of threads

Fig. 1. Weekly analyses on three measures of forum threads created by active and typical users

\subsection{Do Active Users Make Positive Course Contributions?}

Average Votes of Post \& Comment. The analysis for answering research question two is based on votes of posts and comments. The average votes of posts made by active and typical users for the overall course discussion is 1.74 and 1.25 , respectively (the value of a positive vote is considered as +1 , and a negative vote is -1 ). As shown in Figure 2(a), posts made by active users receive more positive votes than typical users in most of weeks, besides week 5 (1.91-1.92). In addition, the average votes of comments created by both groups of users are also studied. For the entire course period, active users' comments received an average 0.64 votes, which is 0.16 higher than typical users. For the 7-week discussion, active users received higher average votes in 5 of the weeks, but comments of typical users have more votes in week 5 and 6 , which are $0.76-0.85$, and 0.69-0.88 of active users-typical users, respectively.

Positive \& Negative Votes in Posts \& Comment. Beside examining average votes of posts and comments, the proportion of posts and comments that include the sum of votes greater or less than 0 is also studied (If the sum of votes in a post/comment is greater than 0 , we consider it generally being a positive vote; otherwise it has a negative vote). For the analysis of posts with positive votes in the overall course period, $45.9 \%$ posts made by active users receive positive votes, which is $7.3 \%$ higher than the posts created by typical users. For the fraction of comments with positive votes in the overall course, active users also have the higher proportion than typical users, which are $37.7 \%$ and 26.6\%, respectively. Moreover, as shown in Figure 2(b) comments made by active users have a higher proportion of positive votes than the comments created by typical users for all the weeks. Considering posts and comments with negative votes, active users have proportions of posts with negative votes as $1.5 \%$ for overall course, which is $1.1 \%$ higher than the posts of typical users. Additionally, the proportion of comments with negative votes made by active users for the entire course is $3.3 \%$ and $1 \%$ for typical users, which are both higher than negative votes of posts (both higher than the proportion of posts). Figure 2(c) shows the weekly analysis of posts and comments with negative votes. Active users have higher proportions of posts with negative vote in almost every week, with a tie in week 4 . In addition, the fractions of comments made by active users having negative votes are higher than those of typical users in most of weeks, except week 1 and week 3 . 


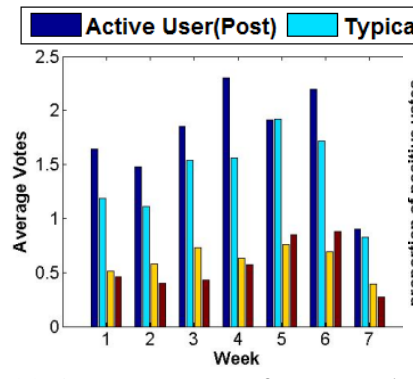

(a) Average votes of posts and (b) comments

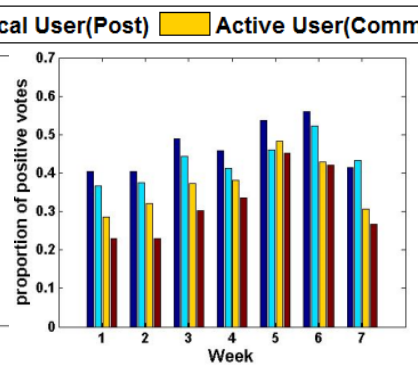

(b) Fraction of posts \& comments with more positive votes

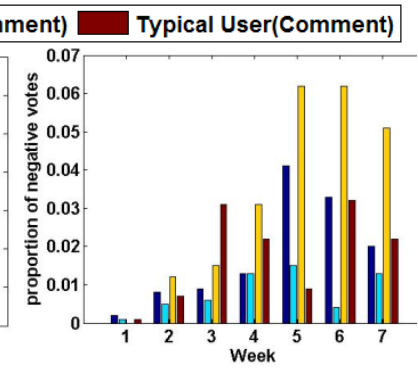

(c) Fraction of posts \& comments with more negative votes

Fig. 2. Weekly analysis on votes of forum posts and comments of active and typical users

\section{Conclusion}

Different analyses on the forum are conducted in this research for providing insightful understanding concerning different users participate in forum discussions. Three measures of threads are analyzed, including the views, replies, and duration of a thread to evaluate whether active users are also more influential users. Based on the analyses of forum threads, active users are also influential users. Furthermore, votes, both positive and negative, of posts and comments are evaluated for identifying whether active users generally make a positive contribution to the forum. Through the overall course period, posts and comments made by active users receive the highest number of votes, on average. For weekly analysis, posts and comments of active users have more votes than typical users in most weeks. However, posts and comments created by active users also have higher probability to receive negative vote than those from typical users. Based on the analysis of votes active, we can still consider that active users generally make a positive contribution to the forum discussion.

\section{References}

1. Smith, M.K., Wood, W.B., Adams, W.K., Wieman, C., Knight, J.K., Guild, N., Su, T.T.: Why peer discussion improves student performance on in-class concept questions. Science 323, 122-124 (2009)

2. Stephens-Martinez, K., Hearst, M.A., Fox, A.: Monitoring moocs: which information sources do instructors value? In: Proceedings of the First ACM Conference on Learning@ Scale Conference, pp. 79-88. ACM (2014)

3. Andresen, M.A.: Asynchronous discussion forums: Success factors, outcomes, assessments, and limitations. Educational Technology \& Society 12, 249-257 (2009)

4. Zhu, E.: Interaction and cognitive engagement: An analysis of four asynchronous online discussions. Instructional Science 34, 451-480 (2006)

5. Wen, M., Yang, D., Rosè, C.P.: Sentiment analysis in MOOC discussion forums: What does it tell us? In: Proceedings of Educational Data Mining (2014)

6. Huang, J., Dasgupta, A., Ghosh, A., Manning, J., Sanders, M.: Superposter behavior in mooc forums. In: Proceedings of the First ACM Conference on Learning@ Scale Conference, pp. 117-126. ACM (2014) 